\title{
Parabolic Ito equations with mixed in time conditions *
}

\author{
Nikolai Dokuchaev \\ Department of Mathematics, Trent University, Ontario, Canada
}

January 30, 2007

\begin{abstract}
We study linear stochastic partial differential equations of parabolic type with special boundary conditions in time. The standard Cauchy condition at the initial time is replaced by a condition that mixes the values of the solution at different times, including the terminal time and continuously distributed times. Uniqueness, solvability and regularity results for the solutions are obtained.
\end{abstract}

AMS 1991 subject classification: Primary 60J55, 60J60, 60H10. Secondary 34F05, 34G10.

Key words and phrases: stochastic partial differential equations, parabolic Ito equations, non-local boundary conditions, non-local in time conditions, mixed in time conditions

\section{Introduction}

Partial differential equations and stochastic partial differential equations (SPDEs) have fundamental significance for natural sciences, and various boundary value problems for them were widely studied. Usually, well-posedness of a boundary value depends on the choice of the boundary value conditions. For the deterministic parabolic equations, well-posedness requires the correct choice of the initial condition. For example, consider the heat equation $u_{t}^{\prime}=u_{x x}^{\prime \prime}, t \in[0, T]$. For this equation, a boundary value problem with the Cauchy condition at initial time $t=0$ is well-posed, and a boundary value problem with the Cauchy condition at terminal time $t=T$ is ill-posed. It is known also that the problems for deterministic parabolic equation are wellposed for periodic type condition $u(x, 0)=u(x, T)$ (see Nakao (1984), Shelukhin (1993), and Dokuchaev (2004)). Less is known for parabolic equation with more general non-local in time conditions and for SPDEs.

\footnotetext{
* Stochastic Analysis and Applications 2008 26, Iss. 3, 562-576.
} 
Boundary value problems for SPDEs are well studied in the existing literature for the case of forward parabolic Ito equations with the Cauchy condition at initial time (see, e.g., Alós et al (1999), Bally et al (1994), Chojnowska-Michalik and Goldys (1995), Da Prato and Tubaro (1996), Gyöngy (1998), Krylov (1999), Maslowski (1995), Pardoux (1993), Rozovskii (1990), Walsh (1986), Zhou (1992), and the bibliography there). Many results have been also obtained for the backward parabolic Ito equations with Cauchy condition at terminal time, as well as for pairs of forward and backward equations with separate Cauchy conditions at initial time and the terminal time respectively (see, e.g., Yong and Zhou (1999), and the author's papers (1992), (2003)). Note that a backward SPDE cannot be transformed into a forward equation by a simple time change, unlike as for the case of deterministic equations. Usually, a backward SPDE is solvable in the sense that there exists a diffusion term being considered as a part of the solution that helps to ensure that the solution is adapted to the driving Brownian motions.

It is, therefore, interesting to extend the existing theory into the problems with conditions that mix the solution at different times in one equality, including initial time and terminal time. The paper investigates these problems for parabolic type SPDEs with the Dirichlet condition at the boundary of the state domain. The standard boundary value Cauchy condition at the initial time is replaces by a condition that mixes in one equation the values of the solution at different times over given time interval, including the terminal time and continuously distributed times. These conditions include only expected values of the solution (see Condition 2.3). For the deterministic case, it covers, in particular, the condition of periodicness and some other non-local boundary value conditions. Uniqueness, existence, and regularity results for the solutions are obtained in $L_{2}$-setting. We found that the solution does not require to include a new diffusion term, in contrast with the case of backward SPDEs, even if the value of the solution at terminal time is involved.

The case of deterministic parabolic equations is also covered, and the results obtained can be still interesting for this simpler case as well.

\section{The problem setting and definitions}

We are given a standard complete probability space $(\Omega, \mathcal{F}, \mathbf{P})$ and a right-continuous filtration $\mathcal{F}_{t}$ of complete $\sigma$-algebras of events, $t \geq 0$. We are given also a $N$-dimensional Wiener process $w(t)$ with independent components; it is a Wiener process with respect to $\mathcal{F}_{t}$.

Assume that we are given an open domain $D \subset \mathbf{R}^{n}$ such that either $D=\mathbf{R}^{n}$ or $D$ is bounded with $C^{2}$-smooth boundary $\partial D$. Let $T>0$ be given, and let $Q \triangleq D \times[0, T]$. 
We will study the following boundary value problem in $Q$

$$
\begin{aligned}
& d_{t} u=(\mathcal{A} u+\varphi) d t+\sum_{i=1}^{N}\left[B_{i} u+h_{i}\right] d w_{i}(t), \quad t \geq 0, \\
& \left.u(x, t, \omega)\right|_{x \in \partial D}=0 \\
& u(x, 0, \omega)-\Gamma u(\cdot)=\xi(x, \omega) .
\end{aligned}
$$

Here $u=u(x, t, \omega), \varphi=\varphi(x, t, \omega), h_{i}=h_{i}(x, t, \omega),(x, t) \in Q, \omega \in \Omega$.

In (2.3), $\Gamma$ is a linear operator that maps functions defined on $Q \times \Omega$ to functions defines on $D \times \Omega . \operatorname{In}(2.1)$,

$$
\mathcal{A} v \triangleq \sum_{i=1}^{n} \frac{\partial}{\partial x_{i}} \sum_{j=1}^{n}\left(b_{i j}(x, t, \omega) \frac{\partial v}{\partial x_{j}}(x)\right)+\sum_{i=1}^{n} f_{i}(x, t, \omega) \frac{\partial v}{\partial x_{i}}(x)+\lambda(x, t, \omega) v(x),
$$

where $b_{i j}, f_{i}, x_{i}$ are the components of $b, f$, and $x$ respectively, and

$$
B_{i} v \triangleq \frac{d v}{d x}(x) \beta_{i}(x, t, \omega)+\bar{\beta}_{i}(x, t, \omega) v(x), \quad i=1, \ldots, N .
$$

We assume that the functions $b(x, t, \omega): \mathbf{R}^{n} \times[0, T] \times \Omega \rightarrow \mathbf{R}^{n \times n}, \beta_{j}(x, t, \omega): \mathbf{R}^{n} \times[0, T] \times \Omega \rightarrow$ $\mathbf{R}^{n}, \bar{\beta}_{i}(x, t, \omega): \mathbf{R}^{n} \times[0, T] \times \Omega \rightarrow \mathbf{R}, f(x, t, \omega): \mathbf{R}^{n} \times[0, T] \times \Omega \rightarrow \mathbf{R}^{n}, \lambda(x, t, \omega): \mathbf{R}^{n} \times[0, T] \times \Omega \rightarrow$ $\mathbf{R}, h_{i}(x, t, \omega): \mathbf{R}^{n} \times[0, T] \times \Omega \rightarrow \mathbf{R}$, and $\varphi(x, t, \omega): \mathbf{R}^{n} \times[0, T] \times \Omega \rightarrow \mathbf{R}$ are progressively measurable with respect to $\mathcal{F}_{t}$ for all $x \in \mathbf{R}^{n}$, and the function $\xi(x, \omega): \mathbf{R}^{n} \times \Omega \rightarrow \mathbf{R}$ is $\mathcal{F}_{0^{-}}$ measurable for all $x \in \mathbf{R}^{n}$. In fact, we will also consider functions $\varphi, \xi$, and $h_{i}$ from wider classes. In particular, we will consider generalized functions $\varphi$.

We do not exclude an important special case when the functions $b, f, \lambda, \varphi$, and $\xi$, are deterministic, and $h_{i} \equiv 0, B_{i} \equiv 0(\forall i)$. In this case, equation (2.1) is deterministic.

\section{Spaces and classes of functions}

We denote by $\|\cdot\|_{X}$ the norm in a linear normed space $X$, and $(\cdot, \cdot)_{X}$ denote the scalar product in a Hilbert space $X$.

We introduce some spaces of real valued functions.

Let $G \subset \mathbf{R}^{k}$ be an open domain, then $W_{q}^{m}(G)$ denote the Sobolev space of functions that belong to $L_{q}(G)$ together with the distributional derivatives up to the $m$ th order, $q \geq 1$.

We denote by $|\cdot|$ the Euclidean norm in $\mathbf{R}^{k}$, and $\bar{G}$ denote the closure of a region $G \subset \mathbf{R}^{k}$.

Let $H^{0} \triangleq L_{2}(D)$, and let $H^{1} \triangleq \stackrel{0}{W_{2}^{1}}(D)$ be the closure in the $W_{2}^{1}(D)$-norm of the set of all smooth functions $u: D \rightarrow \mathbf{R}$ such that $\left.u\right|_{\partial D} \equiv 0$. Let $H^{2}=W_{2}^{2}(D) \cap H^{1}$ be the space equipped with the norm of $W_{2}^{2}(D)$. The spaces $H^{k}$ and $W_{2}^{k}(D)$ are called Sobolev spaces, they are Hilbert spaces, and $H^{k}$ is a closed subspace of $W_{2}^{k}(D), k=1,2$. 
Let $H^{-1}$ be the dual space to $H^{1}$, with the norm $\|\cdot\|_{H^{-1}}$ such that if $u \in H^{0}$ then $\|u\|_{H^{-1}}$ is the supremum of $(u, v)_{H^{0}}$ over all $v \in H^{1}$ such that $\|v\|_{H^{1}} \leq 1 . H^{-1}$ is a Hilbert space.

We shall write $(u, v)_{H^{0}}$ for $u \in H^{-1}$ and $v \in H^{1}$, meaning the obvious extension of the bilinear form from $u \in H^{0}$ and $v \in H^{1}$.

We denote by $\bar{\ell}_{k}$ the Lebesgue measure in $\mathbf{R}^{k}$, and we denote by $\overline{\mathcal{B}}_{k}$ the $\sigma$-algebra of Lebesgue sets in $\mathbf{R}^{k}$.

We denote by $\overline{\mathcal{P}}$ the completion (with respect to the measure $\bar{\ell}_{1} \times \mathbf{P}$ ) of the $\sigma$-algebra of subsets of $[0, T] \times \Omega$, generated by functions that are progressively measurable with respect to $\mathcal{F}_{t}$.

Let $Q_{s} \triangleq D \times[s, T]$. We introduce the spaces

$$
\begin{aligned}
& X^{k}(s, T) \triangleq L^{2}\left([s, T] \times \Omega, \overline{\mathcal{P}}, \bar{\ell}_{1} \times \mathbf{P} ; H^{k}\right), \\
& W_{2}^{k, 0}\left(Q_{s}\right) \triangleq L^{2}\left([s, T], \overline{\mathcal{B}}_{1}, \bar{\ell}_{1} ; H^{k}\right), \\
& Z_{t}^{k} \triangleq L^{2}\left(\Omega, \mathcal{F}_{t}, \mathbf{P} ; H^{k}\right), \\
& \mathcal{C}^{k}(s, T) \triangleq C\left([s, T] ; Z_{T}^{k}\right), \quad k=-1,0,1,2 .
\end{aligned}
$$

The spaces $X^{k}(s, T), W_{2}^{k, 0}(s, T)$, and $Z_{t}^{k}(s, T)$ are Hilbert spaces.

In addition, we introduce the spaces

$$
Y^{k}(s, T) \triangleq X^{k}(s, T) \cap \mathcal{C}^{k-1}(s, T), \quad k=1,2,
$$

with the norm $\|u\|_{Y^{k}(s, T)} \triangleq\|u\|_{X^{k}(s, T)}+\|u\|_{\mathcal{C}^{k-1}(s, T)}$.

For brevity, we shall use the notations $X^{k} \triangleq X^{k}(0, T), \mathcal{C}^{k} \triangleq \mathcal{C}^{k}(0, T), W_{2}^{k, 0}=W_{2}^{k, 0}(Q)$, and $Y^{k} \triangleq Y^{k}(0, T)$.

\section{Conditions for the coefficients}

To proceed further, we assume that Conditions 2.1-2.3 remain in force throughout this paper.

Condition 2.1 The matrix $b=b^{\top}$ is symmetric and bounded. In addition, there exists a constant $\delta>0$ such that

$$
y^{\top} b(x, t, \omega) y-\frac{1}{2} \sum_{i=1}^{N}\left|y^{\top} \beta_{i}(x, t, \omega)\right|^{2} \geq \delta|y|^{2} \quad \forall y \in \mathbf{R}^{n}, \quad(x, t) \in D \times[0, T], \omega \in \Omega .
$$

Inequality (2.6) means that equation (2.1) is superparabolic, in the terminology of Rozovskii (1990).

Condition 2.2 The functions $f(x, t, \omega), \lambda(x, t, \omega), \beta_{i}(x, t, \omega)$, and $\bar{\beta}_{i}(x, t, \omega)$, are bounded. 
Condition 2.3 The operator $\Gamma: Y^{1} \rightarrow Z_{0}^{0}$ is linear, continuous, and such that there exists an integer $m \geq 0$, a set $\left\{t_{i}\right\}_{i=1}^{m} \subset(0, T]$, and linear continuous operators $\bar{\Gamma}_{0}: L_{2}(Q) \rightarrow H^{0}$, $\bar{\Gamma}_{i}: H^{0} \rightarrow H^{0}, i=1, . ., N$, such that

$$
\Gamma u=\mathbf{E}\left\{\bar{\Gamma}_{0} u+\sum_{i=1}^{m} \bar{\Gamma}_{i} u\left(\cdot, t_{i}\right) \mid \mathcal{F}_{0}\right\} .
$$

Moreover, the operators $\bar{\Gamma}_{0}: L_{2}\left([0, T] ; \mathcal{B}_{1}, \ell_{1}, H^{1}\right) \rightarrow W_{2}^{1}(D)$ and $\bar{\Gamma}_{i}: H^{1} \rightarrow W_{2}^{1}(D)$ are continuous.

Condition 2.3 allows to consider $\Gamma$ such that

$$
\bar{\Gamma}_{0} u=\int_{0}^{T} k_{0}(t) u(\cdot, t) d t, \quad \bar{\Gamma}_{i} u\left(\cdot, t_{i}\right)=k_{i} u\left(\cdot, t_{i}\right)
$$

where $k_{0}(\cdot) \in L_{2}(0, T)$ and $k_{i} \in \mathbf{R}$. It covers also $\Gamma$ such that

$$
\bar{\Gamma}_{0} u=\int_{0}^{T} d t \int_{D} k_{0}(x, y, t) u(y, t) d x, \quad \bar{\Gamma}_{i} u\left(\cdot, t_{i}\right)(x)=\int_{D} k_{i}(x, y) u\left(y, t_{i}\right) d y,
$$

where $k_{i}(\cdot)$ are some regular enough kernels.

We introduce the set of parameters

$$
\begin{aligned}
& \mathcal{P} \triangleq(n, D, T, \Gamma, \delta \\
& \operatorname{ess}_{\sup }^{x, t, \omega, i}\left[|b(x, t, \omega)|+|f(x, t, \omega)|+|\lambda(x, t, \omega)|+\left|\beta_{i}(x, t, \omega)\right|+\left|\bar{\beta}_{i}(x, t, \omega)\right|\right] .
\end{aligned}
$$

Sometimes we shall omit $\omega$.

\section{The definition of solution}

Proposition 2.1 Let $\xi \in X^{0}$, let a sequence $\left\{\xi_{k}\right\}_{k=1}^{+\infty} \subset L^{\infty}\left([0, T] \times \Omega, \ell_{1} \times \mathbf{P} ; C(D)\right)$ be such that all $\xi_{k}(\cdot, t, \omega)$ are progressively measurable with respect to $\mathcal{F}_{t}$, and let $\left\|\xi-\xi_{k}\right\|_{X^{0}} \rightarrow 0$. Let $t \in[0, T]$ and $j \in\{1, \ldots, N\}$ be given. Then the sequence of the integrals $\int_{0}^{t} \xi_{k}(x, s, \omega) d w_{j}(s)$ converges in $Z_{t}^{0}$ as $k \rightarrow \infty$, and its limit depends on $\xi$, but does not depend on $\left\{\xi_{k}\right\}$.

Proof follows from completeness of $X^{0}$ and from the equality

$$
\mathbf{E} \int_{0}^{t}\left\|\xi_{k}(\cdot, s, \omega)-\xi_{m}(\cdot, s, \omega)\right\|_{H^{0}}^{2} d s=\int_{D} d x \mathbf{E}\left(\int_{0}^{t}\left(\xi_{k}(x, s, \omega)-\xi_{m}(x, s, \omega)\right) d w_{j}(s)\right)^{2} .
$$

Definition 2.1 Let $\xi \in X^{0}, t \in[0, T], j \in\{1, \ldots, N\}$, then we define $\int_{0}^{t} \xi(x, s, \omega) d w_{j}(s)$ as the limit in $Z_{t}^{0}$ as $k \rightarrow \infty$ of a sequence $\int_{0}^{t} \xi_{k}(x, s, \omega) d w_{j}(s)$, where the sequence $\left\{\xi_{k}\right\}$ is such as in Proposition 2.1. 
Definition 2.2 Let $u \in Y^{1}, \varphi \in X^{-1}$, and $h_{i} \in X^{0}$. We say that equations (2.1)-(2.2) are satisfied if

$$
\begin{aligned}
& u(\cdot, t, \omega)-u(\cdot, r, \omega) \\
& \quad=\int_{r}^{t}(\mathcal{A} u(\cdot, s, \omega)+\varphi(\cdot, s, \omega)) d s+\sum_{i=1}^{N} \int_{r}^{t}\left[B_{i} u(\cdot, s, \omega)+h_{i}(\cdot, s, \omega)\right] d w_{i}(s)
\end{aligned}
$$

for all $r, t$ such that $0 \leq r<t \leq T$, and this equality is satisfied as an equality in $Z_{T}^{-1}$.

Note that the condition on $\partial D$ is satisfied in the sense that $u(\cdot, t, \omega) \in H^{1}$ for a.e. $t, \omega$. Further, $u \in Y^{1}$, and the value of $u(\cdot, t, \omega)$ is uniquely defined in $Z_{T}^{0}$ given $t$, by the definitions of the corresponding spaces. The integrals with $d w_{i}$ in (2.7) are defined as elements of $Z_{T}^{0}$. The integral with $d s$ in (2.7) is defined as an element of $Z_{T}^{-1}$. In fact, Definition 2.2 requires for (2.1) that this integral must be equal to an element of $Z_{T}^{0}$ in the sense of equality in $Z_{T}^{-1}$.

\section{The main results}

Theorem 3.1 There exist a number $\kappa=\kappa(\mathcal{P})>0$ such that problem (2.1)-(2.3) has an unique solution in the class $Y^{1}$, for any $\varphi \in X^{-1}, h_{i} \in X^{0}, \xi \in Z_{0}^{0}$, and any $\Gamma$ such that $\|\Gamma\| \leq \kappa$, where $\|\Gamma\|$ is the norms of the operator $\Gamma: Y^{1} \rightarrow Z_{0}^{T}$. In addition,

$$
\|u\|_{Y^{1}} \leq C\left(\|\varphi\|_{X^{-1}}+\|\xi\|_{Z_{0}^{0}}+\sum_{i=1}^{N}\left\|h_{i}\right\|_{X^{0}}\right),
$$

where $C=C(\kappa, \mathcal{P})>0$ is a constant that depends only on $\kappa$ and $\mathcal{P}$.

Let $\mathbb{I}$ denote the indicator function.

Theorem 3.2 Let $\bar{\Gamma}_{0}$ in Condition 2.3 be such that there exists $\tau>0$ such that $\bar{\Gamma}_{0} u=$ $\bar{\Gamma}_{0}\left(\mathbb{I}_{\{t \geq \tau\}} u\right)$. Then

$$
\|u\|_{Y^{1}} \leq C\left(\|\varphi\|_{X^{-1}}+\|u\|_{X^{-1}}+\|\xi\|_{Z_{0}^{0}}+\sum_{i=1}^{N}\left\|h_{i}\right\|_{X^{0}}\right)
$$

for all solutions $u$ of problem (2.1)-(2.3) in the class $Y^{1}$, where $C=C(\mathcal{P})>0$ is a constant that depends only on $\mathcal{P}$.

Starting from now and up to the end of this section, we assume that Condition 3.1 holds.

Condition 3.1 The domain $D$ is bounded. The functions $b(x, t, \omega), f(x, t, \omega), \lambda(x, t, \omega), \beta_{i}(x, t, \omega)$ and $\bar{\beta}_{i}(x, t, \omega)$ are differentiable in $x$ for a.e. $t, \omega$, and the corresponding derivatives are bounded. 
It follows from this condition that there exists modifications of $\beta_{i}$ such that the functions $\beta_{i}(x, t, \omega)$ are continuous in $x$ for a.e. $t, \omega$. We assume that $\beta_{i}$ are such functions.

Theorem 3.3 Let $\mathcal{F}_{0}$ be the $\mathbf{P}$-augmentation of the set $\{\emptyset, \Omega\}$. Assume that at least one of the following conditions is satisfied:

(i) the function $b$ is non-random, or

(ii) $\beta_{i}(x, t, \omega)=0$ for $x \in \partial D, i=1, \ldots, N$.

Further, assume that problem (2.1)-(2.3) with $\varphi \equiv 0, h_{i} \equiv 0, \xi \equiv 0$, does not admit non-zero solutions in the class $Y^{1}$. Then problem (2.1)-(2.3) has a unique solution $u$ in the class $Y^{1}$ for any $\varphi \in X^{-1}, h_{i} \in X^{0}$, and $\xi \in H^{0}$. In addition,

$$
\|u\|_{Y^{1}} \leq C\left(\|\varphi\|_{X^{-1}}+\|\xi\|_{H^{0}}+\sum_{i=1}^{N}\left\|h_{i}\right\|_{X^{0}}\right),
$$

where $C>0$ is a constant that does not depend on $\varphi, h_{i}$, and $\xi$.

Theorem 3.4 Let the functions $b, f$ and $\lambda$ be non-random and such that the operator $\mathcal{A}$ can be represented as

$$
\mathcal{A} v=\sum_{i, j=1}^{n} \frac{\partial^{2}}{\partial x_{i} \partial x_{j}}\left(b_{i j}(x, t) v(x)\right)+\sum_{i=1}^{n} \frac{\partial}{\partial x_{i}}\left(\widehat{f}_{i}(x, t) p(x)\right)+\widehat{\lambda}(x, t) v(x),
$$

where $\hat{\lambda}(x, t) \leq 0$, and where $\widehat{f}_{i}$ are bounded functions. Further, let

$$
\Gamma u=\mathbf{E}\left\{\int_{0}^{T} k_{0}(t) u(\cdot, t) d t+\sum_{i=1}^{m} k_{i} u\left(\cdot, t_{i}\right) \mid \mathcal{F}_{0}\right\},
$$

where $t_{i}>0$, and where $k_{i} \in \mathbf{R}, k_{0}(\cdot) \in L_{2}(0, T)$ are such that

$$
\int_{0}^{T}\left|k_{0}(t)\right| d t+\sum_{i=1}^{m}\left|k_{i}\right| \leq 1
$$

Then problem (2.1)-(2.3) has a unique solution $u$ in the class $Y^{1}$ for any $\varphi \in X^{-1}, h_{i} \in X^{0}$, and $\xi \in H^{0}$. In addition, (3.3) holds with a constant $C>0$ that does not depend on $\varphi, h_{i}$, and $\xi$.

The following corollary is a special case of Theorem 3.4 for deterministic parabolic equation with the boundary condition that covesr the condition of periodicness. 
Corollary 3.1 Under the assumptions of Theorem 3.4, for any $k \in[-1,1]$, the deterministic boundary value problem

$$
\frac{\partial u}{\partial t}=\mathcal{A} u+\varphi,\left.\quad u\right|_{\partial D}=0, \quad u(x, 0)-k u(x, T) \equiv \Phi(x)
$$

has a unique solution $u \in C\left([0, T] ; H^{0}\right) \cap L_{2}\left([0, t], \mathcal{B}_{1}, \ell_{1}, H^{1}\right)$ for any $\Phi \in H^{0}, \varphi \in L_{2}(Q)$, and

$$
\|u(\cdot, t)\|_{Y^{1}} \leq C\left(\|\Phi\|_{H^{0}}+\|\varphi\|_{L_{2}(Q)}\right)
$$

where $C>0$ is a constant that does not depend on $\Phi$ and $\varphi$.

The classical result about well-posedness of the Cauchy condition at initial time corresponds to the special case of $k=0$.

Corollary 3.1 is close to Theorem 2.2 from Dokuchaev (2004), where boundary value problems for deterministic parabolic equations were studied in a setting that corresponds to the special case when $\Phi=0$; the cited paper was devoted mostly to the case when $T=+\infty$, and the proofs there were based on a different approach.

\section{Proofs}

Let $s \in[0, T), \varphi \in X^{-1}$ and $\Phi \in Z_{s}^{0}$. Consider the problem

$$
\begin{aligned}
& d_{t} u=(\mathcal{A} u+\varphi) d t+\sum_{i=1}^{N}\left[B_{i} u+h_{i}\right] d w_{i}(t), \quad t \geq s, \\
& \left.u(x, t, \omega)\right|_{x \in \partial D}=0, \\
& u(x, s, \omega)=\Phi(x, \omega) .
\end{aligned}
$$

The following lemma represents an analog of the so-called "the first energy inequality", or "the first fundamental inequality" known for deterministic parabolic equations (see, e.g., inequality (3.14) from Ladyzhenskaya (1985), Chapter III).

Lemma 4.1 Assume that Conditions 2.1-2.3 are satisfied. Then problem (4.1) has an unique solution $u$ in the class $Y^{1}(s, T)$ for any $\varphi \in X^{-1}(s, T), h_{i} \in X^{0}(s, T), \Phi \in Z_{s}^{0}$, and

$$
\|u\|_{Y^{1}(s, T)} \leq c\left(\|\varphi\|_{X^{-1}(s, T)}+\|\Phi\|_{Z_{s}^{0}}+\sum_{i=1}^{N}\left\|h_{i}\right\|_{X^{0}(s, T)}\right),
$$

where $c=c(\mathcal{P})$ is a constant that depends on $\mathcal{P}$ only.

(See, e.g., Rozovskii (1990), Chapter 3, Section 4.1). 
Note that the solution $u=u(\cdot, t)$ is continuous in $t$ in $L_{2}\left(\Omega, \mathcal{F}, \mathbf{P}, H^{0}\right)$, since $Y^{1}(s, T)=$ $X^{1}(s, T) \cap \mathcal{C}^{0}(s, T)$.

The following lemma represents an analog of the so-called "the second energy inequality", or "the second fundamental inequality" known for the deterministic parabolic equations (see, e.g., inequality (4.56) from Ladyzhenskaya (1985), Chapter III).

Lemma 4.2 [Dokuchaev (2005)] Assume that Conditions 2.1-2.3, and 3.1, are satisfied. In addition, assume that $\beta_{i}(x, t, \omega)=0$ for $x \in \partial D, i=1, \ldots, N$. Then problem (4.1) has an unique solution $u \in Y^{2}$ for any $\varphi \in X^{0}, h_{i} \in X^{1}, \Phi \in Z_{0}^{1}$, and

$$
\|u\|_{Y^{2}} \leq c\left(\|\varphi\|_{X^{0}}+\|\Phi\|_{Z_{0}^{1}}+\sum_{i=1}^{N}\left\|h_{i}\right\|_{X^{1}}\right),
$$

where $c>0$ is a constant that does not depend on $\varphi, h_{i}$, and $\Phi$.

The constant $C$ in (4.3) depends on $\mathcal{P}$ and some other parameters related to Condition 3.1 (see details in Dokuchaev (2005)).

Introduce operators $L_{s}: X^{-1}(s, T) \rightarrow Y^{1}(s, T)$ and $\mathcal{L}_{s}: Z_{s}^{0} \rightarrow Y^{1}(s, T)$, such that $u=$ $L_{s} \varphi+\mathcal{L}_{s} \Phi+\sum_{i=1}^{N} \mathcal{M}_{i} h_{i}$, where $u$ is the solution in $Y^{1}(s, T)$ of problem (4.1). By Lemma 4.1, these linear operators are continuous.

Let $\bar{X}^{k} \triangleq L^{2}\left([0, T] \times \Omega, \overline{\mathcal{F}_{0} \times \mathcal{B}_{1}}, \bar{\ell}_{1} \times \mathbf{P} ; H^{k}\right)$, where $\overline{\mathcal{F}_{0} \times \mathcal{B}_{1}}$ is the completion (with respect to the measure $\left.\bar{\ell}_{1} \times \mathbf{P}\right)$ of the $\sigma$-algebra of subsets of $[0, T] \times \Omega$, generated by functions that are measurable with respect to $\mathcal{F}_{0}$.

Let $\mathcal{E}_{s}: Z_{T}^{0} \rightarrow Z_{s}^{0}$ be the projector of $Z_{T}^{0}$ on $Z_{s}^{0}$, and let $\mathcal{E}: X_{0} \rightarrow \bar{X}_{0}$ be the projector of $X^{0}$ on $\bar{X}_{0}$.

Introduce operators $\mathcal{Q}: Z_{0}^{0} \rightarrow Z_{0}^{0}$ and $\mathcal{T}_{0}: X^{-1} \rightarrow Z_{0}^{0}, \mathcal{T}_{i}: X^{0} \rightarrow Z_{0}^{0}, i=1, \ldots, N$, such that $\mathcal{Q} \Phi+\mathcal{T}_{0} \varphi+\sum_{i=1}^{N} \mathcal{T}_{i} h_{i}=\Gamma u$, where $u$ is the solution in $Y^{1}$ of problem (4.1) with $s=0$, $\varphi \in X^{-1}, h_{i} \in X^{0}$, and $\Phi \in Z_{0}^{0}$. It is easy to see that these operators are linear and continuous.

Proof of Theorem 3.1. For brevity, we denote $u(\cdot, t)=u(x, t, \omega)$. Clearly, $u \in Y^{1}$ is the solution of problem (2.1)-(2.3), if

$$
\begin{aligned}
& u=L_{0} \varphi+\sum_{i=1}^{N} \mathcal{M}_{i} h_{i}+\mathcal{L}_{0} u(\cdot, 0), \\
& u(\cdot, 0)-\Gamma u=\xi
\end{aligned}
$$

Since $\Gamma u=\mathcal{Q} u(\cdot, 0)+\mathcal{T}_{0} \varphi+\sum_{i=1}^{N} \mathcal{T}_{i} h_{i}$, we have

$$
u=L_{0} \varphi+\sum_{i=1}^{N} \mathcal{M}_{i} h_{i}+\mathcal{L}_{0} u(\cdot, 0),
$$




$$
u(\cdot, 0)-\mathcal{Q} u(\cdot, 0)-\mathcal{T}_{0} \varphi-\sum_{i=1}^{N} \mathcal{T}_{i} h_{i}=\xi .
$$

Clearly, $\|\mathcal{Q}\| \leq\|\Gamma\|\left\|\mathcal{L}_{0}\right\|$, where $\|\mathcal{Q}\|,\|\Gamma\|$, and $\left\|\mathcal{L}_{0}\right\|$, are the norms of the operators $\mathcal{Q}: Z_{0}^{0} \rightarrow$ $Z_{0}^{0}, \Gamma: Y^{1} \rightarrow Z_{0}^{T}$, and $\mathcal{L}_{0}: Z_{0}^{0} \rightarrow Y^{1}$, respectively. Since the operator $\mathcal{Q}: Z_{0}^{0} \rightarrow Z_{0}^{0}$ is continuous, the operator $(I-\mathcal{Q})^{-1}: Z_{0}^{0} \rightarrow Z_{0}^{0}$ is continuous for small enough $\|\mathcal{Q}\|$, i.e. for a small enough $\kappa>0$. Hence

$$
u(\cdot, 0)=(I-\mathcal{Q})^{-1}\left(\xi+\mathcal{T}_{0} \varphi+\sum_{i=1}^{N} \mathcal{T}_{i} h_{i}\right),
$$

and

$$
\begin{aligned}
u & =L_{0} \varphi+\sum_{i=1}^{N} \mathcal{M}_{i} h_{i}+\mathcal{L}_{0} u(\cdot, 0) \\
& =L_{0} \varphi+\sum_{i=1}^{N} \mathcal{M}_{i} h_{i}+\mathcal{L}_{0}(I-\mathcal{Q})^{-1}\left(\xi+\mathcal{T}_{0} \varphi+\sum_{i=1}^{N} \mathcal{T}_{i} h_{i}\right)
\end{aligned}
$$

Then the proof of Theorem 3.1 follows.

Proof of Theorem 3.2. For a real $q>0$, set $u_{q}(x, t, \omega) \triangleq e^{q t} u(x, t, \omega)$. Then $u_{q}$ is the solution of problem (2.1)-(2.3) with $\varphi$ is replaced by $\varphi+q \cdot u$, and with $\lambda$ and $\Gamma$ replaced by $\lambda_{q}$ and $\bar{\Gamma}_{q}$, where

$$
\lambda_{q} \triangleq \lambda+q, \quad \bar{\Gamma}_{q} u=\mathcal{E}_{0}\left\{\bar{\Gamma}_{0 q} u+\sum_{i=1}^{m} \bar{\Gamma}_{i q} u\left(\cdot, t_{i}\right) \mid \mathcal{F}_{0}\right\},
$$

with $\bar{\Gamma}_{0 q} u=\bar{\Gamma}_{0}\left(e^{-q t} u\right), \bar{\Gamma}_{i q} u\left(\cdot, t_{i}\right)=\bar{\Gamma}_{i}\left(e^{-q t_{i}} u\left(\cdot, t_{i}\right)\right)$. By the assumptions on $\bar{\Gamma}_{0}$ and by the choice of $t_{i}>0$, we have that $\left\|\bar{\Gamma}_{q}\right\| \rightarrow 0$ as $q \rightarrow+\infty$, for the norm of the operator $\bar{\Gamma}_{q}: Y^{1} \rightarrow Z_{0}^{T}$. By Lemma 4.1 and Theorem 3.1, it follows that, for a large $q>0$,

$$
\left\|u_{q}\right\|_{Y^{1}} \leq C\left(\|\varphi\|_{X^{-1}}+\left\|u_{q}\right\|_{X^{-1}}+\|\xi\|_{H_{0}}+\sum_{i=1}^{N}\left\|h_{i}\right\|_{X^{0}}\right),
$$

where $C=C(q, \mathcal{P})$ is a constant that does not depend on $u, \varphi, h_{i}, \xi$. Then the proof of Theorem 3.2 follows.

Starting from now, we assume that Condition 3.1 is satisfied.

Lemma 4.3 Let $\mathcal{F}_{0}$ be the $\mathbf{P}$-augmentation of the set $\{\emptyset, \Omega\}$, and let the function $b$ be deterministic. Then the operator $\mathcal{Q}: Z_{0}^{0} \rightarrow Z_{0}^{0}$ is compact.

Proof of Lemma 4.3. Consider the following auxiliary boundary value problem:

$$
\begin{aligned}
& \frac{d \bar{u}}{d t}=\overline{\mathcal{A}} \bar{u}+\bar{\eta}, \quad t>s, \\
& \left.\bar{u}\right|_{x \in \partial D}=0, \quad \bar{u}(x, s)=\Phi(x),
\end{aligned}
$$


where $\bar{\eta} \in W_{2}^{-1,0}, \Phi \in H^{0}$ and

$$
\overline{\mathcal{A}} v \triangleq \sum_{i, j=1}^{n} b_{i j}(x, t) \frac{\partial^{2} v}{\partial x_{i} \partial x_{j}}(x)+\frac{\partial v}{\partial x}(x) \mathbf{E} f(x, t, \omega)+v(x) \mathbf{E} g(x, t, \omega) .
$$

From the first energy inequality (3.14) from Ladyzhenskaya (1985), Chapter III, it follows that

$$
\|\bar{u}(\cdot, T)\|_{H^{0}}+\|\bar{u}\|_{W_{2}^{1,0}\left(Q_{s}\right)} \leq C_{1}\left(\|\bar{u}(\cdot, s)\|_{H^{0}}+\|\bar{\eta}\|_{W_{2}^{-1,0}\left(Q_{s}\right)}\right),
$$

where $C_{1}=C_{1}(\mathcal{P})>0$ is a constant that does not depend on $\Phi$ and $s$.

Introduce operators $\bar{L}_{s}: W_{2}^{-1,0}\left(Q_{s}\right) \rightarrow W_{2}^{1,0}\left(Q_{s}\right)$ and $\overline{\mathcal{L}}_{s}: H^{0} \rightarrow W_{2}^{1,0}\left(Q_{s}\right)$, such that $\bar{u}=\bar{L}_{s} \bar{\eta}+\overline{\mathcal{L}}_{s} \Phi$, where $\bar{u}$ is the solution of problem (4.5). By (4.6), these linear operators are continuous.

Let $u \triangleq \mathcal{L}_{0} \Phi$, where $\Phi \in H^{0}$, and let $\bar{u} \triangleq \mathcal{E} u$. This $\bar{u}$ is the solution of problem (4.5) with

$$
\bar{\eta}=\mathbf{E}\left(\frac{\partial u}{\partial x}(x, t, \omega) f(x, t, \omega)+g(x, t, \omega) u(x, t, \omega)\right)-\frac{\partial \bar{u}}{\partial x}(x, t) \mathbf{E} f(x, t, \omega)-\bar{u}(x, t) \mathbf{E} g(x, t, \omega) .
$$

It is easy to see that there exist constants $C_{i}=C_{i}(\mathcal{P})>0, i=1,2$, which do not depend on $\Phi$ and such that

$$
\|\bar{\eta}\|_{L_{2}\left(Q_{s}\right)} \leq C_{1}\|u\|_{X^{1}} \leq C_{2}\|\Phi\|_{H^{0}} .
$$

By the second energy inequality (4.56) from Ladyzhenskaya (1985), Chapter III, it follows that

$$
\|\bar{u}(\cdot, \tau)\|_{H^{1}}+\|\bar{u}\|_{W_{2}^{2,0}\left(Q_{s}\right)} \leq C_{*}\left(\|\bar{u}(\cdot, s)\|_{H^{1}}+\|\bar{\eta}\|_{L_{2}\left(Q_{s}\right)}\right), \quad \tau \in[s, T],
$$

where $C_{*}>0$ is a constant that does not depend on $\bar{u}, \bar{\eta}, s$, and $\tau$.

We have that $\left.\bar{u}\right|_{t \in[s, T]}=\left.\bar{L}_{s} \bar{\eta}\right|_{t \in[s, T]}+\overline{\mathcal{L}}_{s} \bar{u}(\cdot, s)$ for all $s \in[0, T]$, and, for $\tau \in\left\{t_{1}, \ldots, t_{m}\right\}$,

$$
\begin{aligned}
\left\|\bar{\Gamma}_{i} u(\cdot, \tau)\right\|_{W_{2}^{1}(D)}^{2} & \leq C_{0}\|\bar{u}(\cdot, \tau)\|_{H^{1}}^{2} \leq C_{1}\left(\inf _{t \in[0, \tau]}\|\bar{u}(\cdot, t)\|_{H^{1}}^{2}+\|\bar{\eta}\|_{L_{2}\left(Q_{\tau}\right)}^{2}\right) \\
& \leq \frac{C_{1}}{\tau} \int_{0}^{\tau}\|\bar{u}(\cdot, t)\|_{H^{1}}^{2} d t+C_{1}\|\bar{\eta}\|_{L_{2}(Q)}^{2} \leq \frac{C_{2}}{\tau}\|u\|_{X^{1}}^{2} \leq \frac{C_{3}}{\tau}\|\Phi\|_{H^{0}}
\end{aligned}
$$

for constants $C_{i}>0$ that do not depend on $\Phi$. In addition,

$$
\left\|\bar{\Gamma}_{0} u\right\|_{W_{2}^{1}(D)}^{2} \leq c_{0} \int_{0}^{T}\|\bar{u}(\cdot, t)\|_{H^{1}}^{2} d t \leq c_{1}\|\Phi\|_{H^{0}}
$$

for constants $c_{i}=c_{i}(\mathcal{P})>0$ that do not depend on $\Phi$. Hence the operator $\mathcal{Q}: H^{0} \rightarrow H^{1}$ is continuous. The embedding of $W_{2}^{1}(D)$ into $H^{0}$ is a compact operator (see, e.g., Theorem 7.3 from Ladyzhenskaia (1985), Chapter I). Then the proof of Lemma 4.3 follows.

Lemma 4.4 Let $\mathcal{F}_{0}$ be the $\mathbf{P}$-augmentation of the set $\{\emptyset, \Omega\}$, and let $\left.\beta_{i}(x, t, \omega)\right|_{x \in \partial D}=0$ for $i=1, \ldots, N$. Then the operator $\mathcal{Q}: Z_{0}^{0} \rightarrow Z_{0}^{0}$ is compact. 
Proof of Lemma 4.4. Set $u=\mathcal{L}_{0} \Phi$, where $\Phi \in H^{0}$. We have that $\left.u\right|_{t \in[s, T]}=\mathcal{L}_{s} u(\cdot, s)$ for all $s \in[0, T]$. By Lemmas 4.1 and 4.2 , we have for $\tau \in\left\{t_{1}, \ldots, t_{m}\right\}$ that

$$
\begin{aligned}
& \left\|\bar{\Gamma}_{i} u(\cdot, \tau)\right\|_{W_{2}^{1}(D)}^{2} \leq C_{0}\|u(\cdot, \tau)\|_{Z_{0}^{1}}^{2} \leq C_{1} \inf _{t \in[0, \tau]}\|u(\cdot, t)\|_{Z_{t}^{1}}^{2} \leq \frac{C_{1}}{\tau} \int_{0}^{\tau}\|u(\cdot, t)\|_{Z_{t}^{1}}^{2} d t \leq \frac{C_{3}}{\tau}\|\Phi\|_{H^{0}}^{2}, \\
& \left\|\bar{\Gamma}_{0} u\right\|_{W_{2}^{1}(D)}^{2} \leq C_{4} \int_{0}^{T}\|u(\cdot, t)\|_{H^{1}}^{2} d t \leq C_{5}\|\Phi\|_{H^{0}}
\end{aligned}
$$

for constants $C_{i}>0$ which do not depend on $\Phi$. Hence the operator $\mathcal{Q}: H^{0} \rightarrow H^{1}$ is continuous. Since the embedding of $W_{2}^{1}(D)$ to $H^{0}$ is a compact operator, the proof of Lemma 4.3 follows.

Proof of Theorem 3.3. By the assumptions, the equation $\mathcal{Q} \Phi=\Phi$ has the only solution $\Phi=0$ in $H^{0}$. By Lemmas 4.3-4.4 and by the Fredholm Theorem, the operator $(I+\mathcal{Q})^{-1}: H^{0} \rightarrow H^{0}$ is continuous. Then the proof of Theorem 3.3 follows from representation (4.4).

Proof of Theorem 3.4. It suffices to show that the assumptions of Theorem 3.3 are satisfied. For $\eta \in W_{2}^{-1,0}$ and $\Phi \in H^{0}$, consider the problem

$$
\begin{aligned}
& \frac{d \bar{u}}{d t}=\mathcal{A} \bar{u}+\eta, \quad t>s, \\
& \left.\bar{u}\right|_{x \in \partial D}=0, \quad \bar{u}(x, s)=\Phi(x) .
\end{aligned}
$$

We will use the operators $\bar{L}_{s}: W_{2}^{-1,0}\left(Q_{s}\right) \rightarrow W_{2}^{1,0}\left(Q_{s}\right)$ and $\overline{\mathcal{L}}_{s}: H^{0} \rightarrow W_{2}^{1,0}\left(Q_{s}\right)$, such that $\bar{u}=\bar{L}_{s} \eta+\overline{\mathcal{L}}_{s} \Phi$, where $\bar{u}$ is the solution of problem (4.8). By (4.6), these linear operators are continuous, as well as the operators $\bar{L}_{s}: W_{2}^{-1,0}\left(Q_{s}\right) \rightarrow C\left([s, T] ; H^{0}\right)$ and $\overline{\mathcal{L}}_{s}: H^{0} \rightarrow$ $C\left([s, T] ; H^{0}\right)$.

Let $u=\mathcal{E} \mathcal{L}_{0} u(\cdot, 0)$ and $\bar{u} \triangleq \mathcal{E} u$. Then $\bar{u}=\overline{\mathcal{L}}_{0} u(\cdot, 0)$.

Let

$$
\zeta^{+}(x) \triangleq \max (0, \bar{u}(x, 0)), \quad \zeta^{-}(x) \triangleq \max (0,-u(x, 0)), \quad \bar{u}^{-\triangleq} \triangleq \overline{\mathcal{L}}_{0} \zeta^{-}, \quad \bar{u}^{+} \triangleq \overline{\mathcal{L}}_{0} \zeta^{+} .
$$

Since $\bar{u}(x, 0) \equiv \zeta^{+}(x)-\zeta^{-}(x)$, we have that $\bar{u}^{+} \geq 0, \bar{u}^{-} \geq 0$, and $\bar{u}=\bar{u}^{+}-\bar{u}^{-}$a.e..

Let $\nu^{ \pm} \triangleq \int_{D} \zeta^{ \pm}(x) d x$. If $\bar{u}(\cdot, 0) \neq 0$ then either $\zeta^{+}(\cdot, 0) \neq 0$ or $\zeta^{-}(\cdot, 0) \neq 0$, i.e., either $\nu^{+} \neq 0$ or $\nu^{-} \neq 0$. Clearly, if $\nu^{ \pm} \neq 0$, then $\bar{u}^{ \pm} / \nu^{ \pm}$can be represented as the probability density function of a diffusion process being absorbed at $\partial D$ and being killed inside $D$ with the rate $\widehat{\lambda} \geq 0$. The absorption at $\partial D$ causes that if $\zeta^{ \pm}(\cdot, 0) \neq 0$ then

$$
\int_{D} \bar{u}^{ \pm}(x, t) d x<\int_{D} \zeta^{ \pm}(x) d x, \quad t>0 .
$$

Hence

$$
\int_{D}|\bar{u}(x, t)| d x=\int_{D} u^{+}(x, t) d x+\int_{D} u^{-}(x, t) d x<\int_{D} u^{+}(x, 0) d x+\int_{D} u^{-}(x, 0) d x=\int_{D}|\bar{u}(x, 0)| d x
$$


for all $t>0$. Hence

$$
\begin{aligned}
\int_{D}|\Gamma \bar{u}(x)| d x & \leq \int_{D}\left|\int_{0}^{T} k_{0}(t) \bar{u}(x, t) d t+\sum_{i=1}^{m} k_{i} \bar{u}\left(x, t_{i}\right)\right| d x \\
& <\left(\int_{0}^{T}\left|k_{0}(t)\right| d t+\sum_{i=1}^{m}\left|k_{i}\right|\right) \int_{D}|\bar{u}(x, 0)| d x \leq \int_{D}|u(x, 0)| d x .
\end{aligned}
$$

Remind that $u(x, 0) \equiv \bar{u}(x, 0)$ and $\Gamma \bar{u}=\Gamma u$. Therefore, the condition $u(x, 0)=\Gamma u$ fails to be satisfied for $u \neq 0$. Thus, $u=0$ is the unique solution of problem (2.1)-(2.3) for $\xi=0, \varphi=0$, and $h_{i}=0$. Then the proof of Theorem 3.4 follows from Lemma 4.3 and from the Fredholm Theorem.

Corollary 3.1 follows immediately from Theorem 3.4.

\section{References}

Alós, E., León, J.A., Nualart, D. (1999). Stochastic heat equation with random coefficients Probability Theory and Related Fields 115, 1, 41-94.

Bally, V., Gyongy, I., Pardoux, E. (1994). White noise driven parabolic SPDEs with measurable drift. Journal of Functional Analysis 120, 484 - 510.

Chojnowska-Michalik, A., and Goldys, B. (1965). Existence, uniqueness and invariant measures for stochastic semilinear equations in Hilbert spaces, Probability Theory and Related Fields, 102, No. 3, 331-356.

Da Prato, G., and Tubaro, L. (1996). Fully nonlinear stochastic partial differential equations, SIAM Journal on Mathematical Analysis 27, No. 1, 40-55.

Dokuchaev, N.G. (1992). Boundary value problems for functionals of Ito processes, Theory of Probability and its Applications 36 , 459-476.

Dokuchaev, N.G. (2003). Nonlinear parabolic Ito's equations and duality approach, Theory of Probability and its Applications 48 (1), 45-62.

Dokuchaev, N.G. (2004). Estimates for distances between first exit times via parabolic equations in unbounded cylinders. Probability Theory and Related Fields, 129, 290 - 314.

Dokuchaev, N.G. (2005). Parabolic Ito equations and second fundamental inequality. Stochastics $\mathbf{7 7}$ (2005), iss. 4., pp. 349-370.

Gyöngy, I. (1998). Existence and uniqueness results for semilinear stochastic partial differential equations. Stochastic Processes and their Applications 73 (2), 271-299.

Krylov, N. V. (1999). An analytic approach to SPDEs. Stochastic partial differential equations: six perspectives, 185-242, Mathematical Surveys and Monographs, 64, AMS., Providence, 
RI, pp.185-242.

Ladyzhenskaia, O.A. (1985). The Boundary Value Problems of Mathematical Physics. New York: Springer-Verlag.

Maslowski, B. (1995). Stability of semilinear equations with boundary and pointwise noise, Annali della Scuola Normale Superiore di Pisa - Classe di Scienze (4), 22, No. 1, 55-93.

Nakao, M. (1984). Periodic solution of some nonlinear degenerate parabolic equations. Journal of Mathematical Analysis and Applications 104 (2), 554-557.

Pardoux, E. (1993). Bulletin des Sciences Mathematiques, 2e Serie, 117, 29-47.

Rozovskii, B.L. (1990). Stochastic Evolution Systems; Linear Theory and Applications to Non-Linear Filtering. Kluwer Academic Publishers. Dordrecht-Boston-London.

Shelukhin, V.V. (1993). Variation principle for non-local in time problems for linear evolutionary equations. Siberian Math. J. 34 (2), 191-207.

Walsh, J.B. (1986). An introduction to stochastic partial differential equations, Lecture Notes in Mathematics 1180, Springer Verlag.

Yong, J., and Zhou, X.Y. (1999). Stochastic controls: Hamiltonian systems and HJB equations. New York: Springer-Verlag.

Zhou, X.Y. (1992). A duality analysis on stochastic partial differential equations, Journal of Functional Analysis 103, No. 2, 275-293. 